\title{
Quantum mechanical complementarity probed in a closed-loop Aharonov-Bohm interferometer
}

\author{
DONG-IN CHANG'1 , GYONG LUCK KHYMM ${ }^{1}, 2$, KICHEON KANG², YUNCHUL CHUNG ${ }^{3 *}$, HU-JONG LEE',4*, \\ MINKY SEO ${ }^{3}$, MOTY HEIBLUMI5 ${ }^{5}$, DIANA MAHALU ${ }^{5}$ AND VLADIMIR UMANSKY ${ }^{5}$ \\ ${ }^{1}$ Department of Physics, Pohang University of Science and Technology, Pohang 790-784, Republic of Korea \\ ${ }^{2}$ Department of Physics, Chonnam National University, Gwangju 500-757, Republic of Korea \\ ${ }^{3}$ Department of Physics, Pusan National University, Busan 609-735, Republic of Korea \\ ${ }^{4}$ National Center for Nanomaterials Technology, Pohang 790-784, Republic of Korea \\ ${ }^{5}$ Braun Center for Submicron Research, Department of Condensed Matter Physics, Weizmann Institute of Science, Rehovot 76100, Israel \\ *e-mail: ycchung@pusan.ac.kr; hjlee@postech.ac.kr
}

The complementarity principle ${ }^{1}$ demands that a particle reveals wave-like properties only when the different paths that it can take are indistinguishable $e^{2,3}$. The complementarity has been demonstrated in optics with pairs of correlated photons $s^{4,5}$ and in two-path solid-state interferometers with phase-coherent electrons ${ }^{6}$. In the latter experiment, a charge detector embedded near one path of a two-path electron interferometer provided which-path information ${ }^{3}$. Here, we report on electron dephasing in an Aharonov-Bohm ring interferometer ${ }^{7}$ via a charge detector adjacent to the ring. In contrast to the two-path interferometer, charge detection in the ring does not always provide path information. The interference was suppressed only when path information could be acquired, even if only in principle. This confirms that dephasing is not always induced by 'disturbing' the interfering particle through the interferometerenvironment interactions: path information of the particle must be available too. Our experiment suggests that acquisition of which-path information is more fundamental than the backaction in understanding quantum mechanical complementarity.

The previous electronic 'which-path' experiments ${ }^{6}$ were based on a solid-state two-path interferometer, fabricated in the plane of a high-mobility two-dimensional electron gas (2DEG). The interferometer consisted of an open Aharonov-Bohm ring, with a source and a drain of electrons weakly coupled to the open ring. In one path of the interferometer, a coherent quantum dot was embedded $^{6-8}$, being electrostatically coupled to a quantum-pointcontact (QPC) charge detector (in the immediate proximity to the quantum dot). An electron trapped in the quantum dot modified the conductance of the nearby QPC and thus enabled charge detection by the $\mathrm{QPC}^{9-11}$. Being an open geometry, with multiple grounded drains (bases) along the paths of the electron, assured that only two paths interfered while the backscattered electrons were drained out by the grounded bases. Thus, the detection of a charge inside the quantum dot (by the QPC) provided path information, which led to the suppression of the Aharonov-Bohm interference oscillations.
Here, we used a 'closed-loop' Aharonov-Bohm interferometer ${ }^{7,12}$, as shown in Fig. 1a, with a quantum dot and a QPC detector placed in a similar manner as in Buks and co-workers ${ }^{6}$. However, in contrast to the previous schemes, the closed geometry allowed an electron to encircle the interferometer loop many times before it reached the drain, making the interferometer an analogue of the Fabry-Perot interferometer, where in the closed ring the forward propagating and the backward propagating paths are spatially separated. Let us look at a couple of examples: among various possible electron trajectories contributing to the interference, the most probable trajectories, which lead to source-drain conductance oscillations with periods of one ( $h / e$; first harmonic) and a half $(h / 2 e$; second harmonic) flux quantum, are illustrated in Fig. $1 \mathrm{~b}$ and $c$, respectively ${ }^{13,14}$. In principle, there are an infinite number of other possible trajectories that give rise to the first- and the second-harmonic interferences. However, as will be discussed below, these two sets of trajectories (shown in Fig. 1b,c) are the dominant ones. For the trajectories plotted in Fig. 1b, path information can be acquired by detecting the presence of an added electron inside the quantum dot. Alternatively, for the trajectories plotted in Fig. 1c, charge detection in the quantum dot does not, in general, provide path information to distinguish between the blue and red trajectories, as both pass the quantum dot once ${ }^{12}$. Hence, we would expect strong dephasing of the first Aharonov-Bohm harmonic but not of the second harmonic. Adding an element of time in the detection process may distinguish between trajectories of an electron trapped in the quantum dot, and also suppress the second harmonics-as we describe below-making this experiment clearly different to its predecessors.

Our closed-loop interferometer (Fig. 1a) was fabricated in a $\mathrm{GaAs} / \mathrm{AlGaAs}$ heterojunction wafer containing a 2DEG with an elastic mean free path of $l_{\mathrm{e}} \sim 20 \mu \mathrm{m}$. Four side gates $\left(\mathrm{M}_{1}, \mathrm{M}_{2}, \mathrm{M}_{3}\right.$, $\mathrm{M}_{4}$ ) together with an island gate (P) defined an Aharonov-Bohm ring of about $550 \mathrm{~nm}$ in radius (Fig. 1a), with approximately five conducting channels $(N>5)$ in each path. A quantum dot and 
a

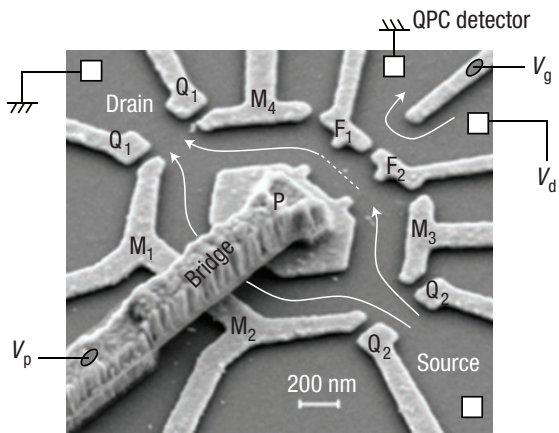

b

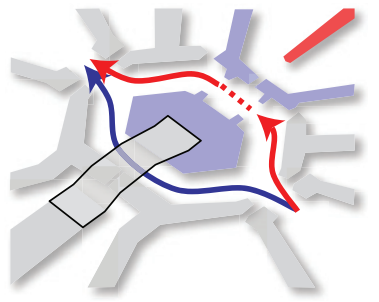

First harmonic (h/e oscillation) path information

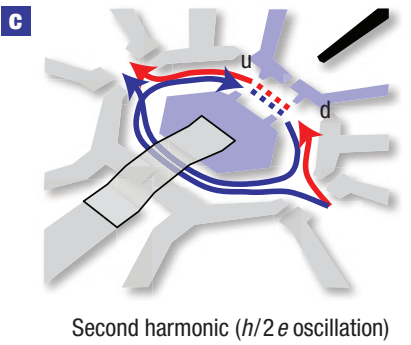

no path information
Figure 1 Which-path interferometer. a, SEM image of a closed-loop Aharonov-Bohm interferometer fabricated on the surface of a 2DEG wafer. The device consists of two parts: an electronic interferometer and a QPC detector. $\mathbf{b}$, The trajectory leading to the first-harmonic interference ( $h$ / $e$ conductance oscillation), where the charge detection is equivalent to the path detection. c, The trajectory leading to the second-harmonic interference ( $h / 2 e$ conductance oscillation), where the charge detection is not necessarily the path detection.

a QPC detector were placed to detect charges trapped inside the quantum dot.

To examine the characteristics of the quantum dot, the left path was pinched off by applying a large negative voltage to the side gates $\mathrm{M}_{1}$ and $\mathrm{M}_{2}$. The Coulomb-blockaded conductance peaks of the quantum dot were monitored by varying the voltage on the centre island gate (supplied by the air bridge P, see Figs 1a and 2a). The quantum dot was tuned to the Coulomb-blockaded conductance peak marked by the vertical arrow in Fig. 2a. The transmission probability of the QPC detector, defined as $T_{\mathrm{d}}=G_{\mathrm{d}} /\left(2 e^{2} / h\right)$, was set to $T_{\mathrm{d}}=0.1775$, where the dephasing rate was found to be the highest ${ }^{6}$. The close proximity between the QPC detector and the quantum dot lead to a strong modification of its transmission, $\Delta T_{\mathrm{d}}$, as illustrated in Fig. 2a, each time the number of electrons inside the quantum dot changed by one. Equivalently, the mere possibility of measuring the added charge to the dot, leads, by the inadvertent back-action of the detector on the quantum dot, to dephasing of the dwelling electron in the quantum dot. Following the above-described tuning of the dot, the current through the detector was shut off and the left arm of the interferometer was opened. The interferometer was then tuned to exhibit both the first and the second harmonic of the Aharonov-Bohm interference.

The effect of the charge (or path) detection on the interference pattern, at different detector bias voltages $(0-400 \mu \mathrm{V})$, is shown in Fig. 2b. The Aharonov-Bohm oscillations clearly exhibit two harmonics (with the field periodicity of $\sim 4.6$ and $\sim 2.3 \mathrm{mT}$ ), which are being suppressed with increasing the bias of the detector, as expected. As the number of 'detecting electrons' that pass the detector during the dwelling of the electrons in the quantum dot
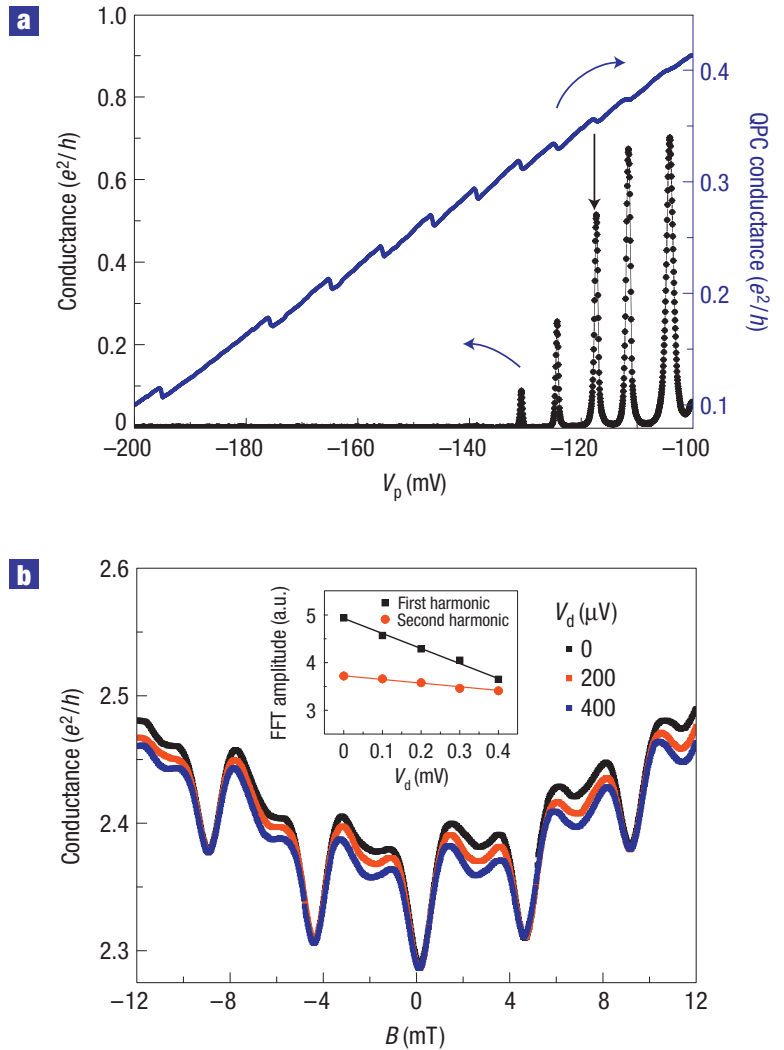

Figure 2 Detection procedure and Aharonov-Bohm oscillation. a, With the left path in Fig. 1a pinched off, the Coulomb blockade of the quantum dot (left vertical axis) and the conductance of the QPC detector (right vertical axis) are taken by sweeping the island gate voltage $\left(V_{p}\right)$. Each time an electron passes through the quantum dot, the transmission through the QPC detector is affected, showing a saw-tooth-like behaviour. $\mathbf{b}$, With both the left and the right paths open, the coherent transmission of the partial waves leads to Aharonov-Bohm oscillations. Around the Coulomb-blockade peak denoted by an arrow in a, Aharonov-Bohm oscillations reveal both the first- and the second-harmonic interferences, where the amplitude of the first harmonic is suppressed with increasing QPC detector bias. However, the second-harmonic amplitude is almost insensitive to the detector bias in this low bias range up to $400 \mu \mathrm{V}$ (also see the inset).

increases, dephasing is enhanced. The inset of Fig. $2 \mathrm{~b}$ clearly shows that the first Aharonov-Bohm harmonic is much more sensitive to the bias of the detector than the second harmonic. As alluded to above, the difference arises from the fact that the charge detection cannot distinguish between the two types of trajectory illustrated in Fig. 1c leading to the second harmonic.

To be more specific, there are two major sets of trajectories for the second harmonic. In the first set of trajectories shown in Fig. 1c, the partial wave of an electron starting with the left path at the source makes one and a half clockwise turns around the interferometer (blue), whereas that starting with the right path makes just a half turn (red). In the second set of trajectories (not shown), the directions of the two partial waves are switched: the shorter path goes though the left and the anticlockwise path makes one and a half turns passing through the quantum dot twice. In the latter case, path information is obtained via charge detection because only the longer path passes through the quantum dot (even twice), whereas the other path never passes through the quantum dot. The transmission probability through the quantum 

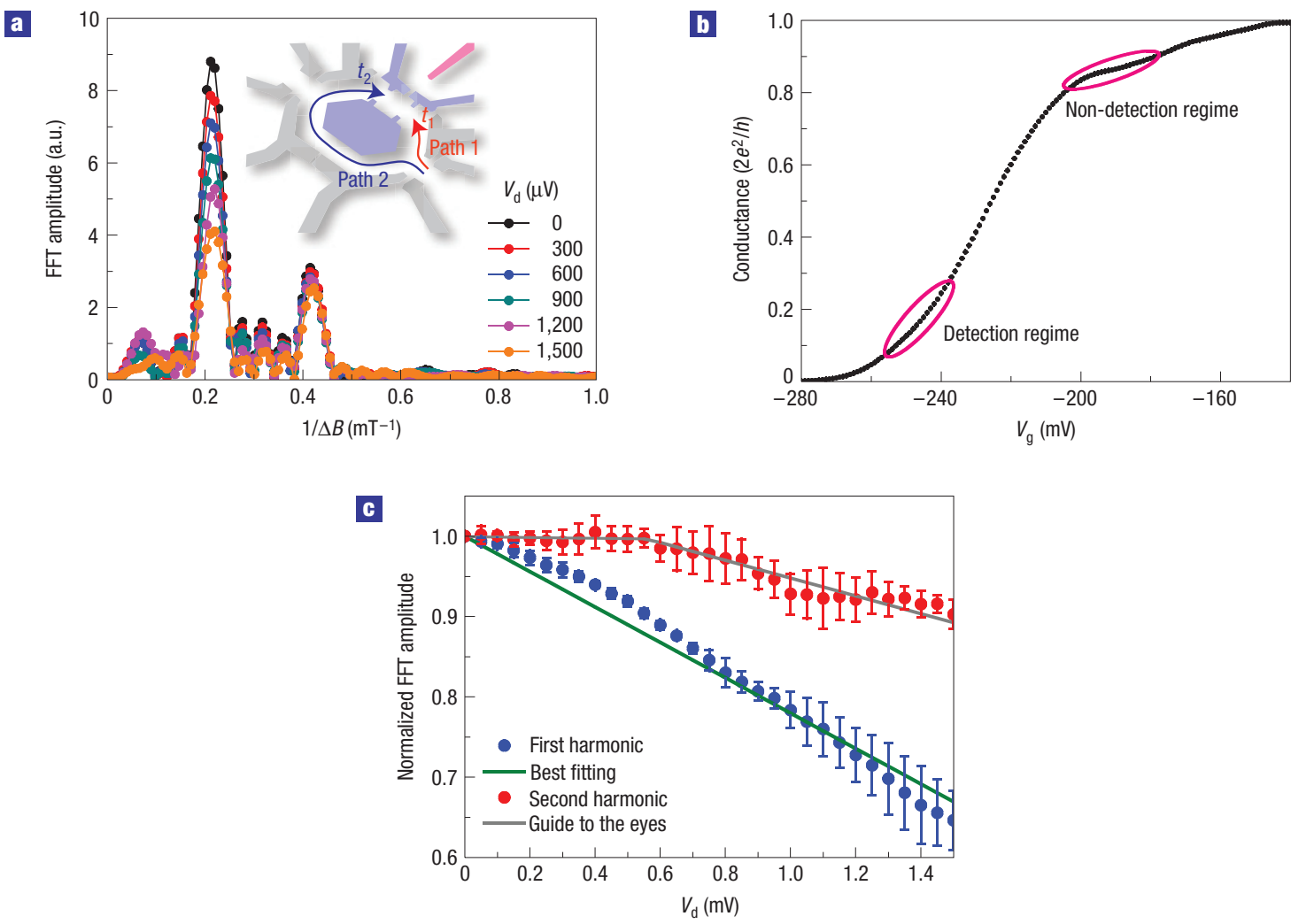

Figure 3 Time-resolving measurements on the second-harmonic interference. a, FFT amplitudes of Aharonov-Bohm oscillations for the first- and the second-harmonic interference with varying the bias of the QPC detector. Inset: Schematic diagram illustrating the source-quantum-dot transit time difference of electron wave packets between the left $\left(t_{2}\right)$ and the right $\left(t_{1}\right)$ paths. The source-quantum-dot distances of the left and right paths are $\sim 2.5 \mu \mathrm{m}$ and $\sim 0.83 \mu \mathrm{m}$, respectively. $\mathbf{b}$, The gate voltage $\left(V_{g}\right)$ dependence of the QPC transmittance showing the least detection-sensitive region (non-detection regime) and the most sensitive region (detection regime). c, FFT amplitudes for the two harmonics normalized by the corresponding zero-detector-bias values. An error bar for each QPC detector bias was determined by the standard deviation for four different sets of measurements.

dot, $T_{\mathrm{P}}$, can be estimated as follows: as the conductance peak is about $G_{\mathrm{QD}} /\left(2 e^{2} / h\right) \sim 0.25$ and the number of transverse channels in each path is about $N \sim 5$, the transmission probability is $T_{\mathrm{P}}=G_{\mathrm{QD}} /\left(2 e^{2} / h\right) / N \sim 0.05$. Therefore, other more complicated sets of trajectories can be ignored owing to the low transmission probability through the quantum dot. This also explains the absence of higher-order harmonics with $n>2$. Furthermore, the second set of trajectories, compared with the first set shown in Fig. 1c, can also be neglected owing to the large dwell time in the quantum dot $\left(t_{\mathrm{d}} \sim 2 \mathrm{~ns}\right)$, which is much larger than the characteristic mean time interval, $t_{\mathrm{f}}$, between successive injection of electrons through the left (shorter) path. From the bias voltage of the interferometer, $V=10 \mu \mathrm{V}$, we find that $t_{\mathrm{f}} \sim h / 2 \mathrm{eV} \sim 0.2 \mathrm{~ns}$. For the first set of trajectories shown in Fig. 1c, both the partial waves pass the quantum dot only once, and there is no considerable time delay in the arrival of the two wave packets. However, for the second set of trajectories, the characteristic time of the shorter path is about $t_{\mathrm{f}} \sim 0.2 \mathrm{~ns}$ and that of the longer path is about $2 t_{\mathrm{d}} \sim 4 \mathrm{~ns}$. This large time delay strongly suppresses the interference owing to the lack of overlap of the wave packets taking two different paths. Therefore, the second harmonic is dominated by the trajectories shown in Fig. 1c, which do not provide path information in the charge detection process.

It should be noted that the sets of electron trajectories such as those described in Fig. 1c may be distinguished via charge detection in the quantum dot if the difference in their dwell time in the interferometer can be differentiated, hence dephasing also the second Aharonov-Bohm harmonic. The difference in the path lengths of the two possible trajectories shown in Fig. 1c, going from the source to the quantum dot, is about $1.7 \mu \mathrm{m}$, and for a Fermi velocity of $v_{\mathrm{F}} \sim 1.62 \times 10^{5} \mathrm{~m} \mathrm{~s}^{-1}$ we get a time difference of $\sim 10$ ps. This must be compared with the time difference between consecutive electron arrivals in the QPC detector, $h / 2 e V_{\mathrm{d}}$, where $V_{\mathrm{d}}$ is the applied bias voltage to the QPC detector ${ }^{6}$. This simple argument leads to the conclusion that path information for the trajectories shown in Fig. 1c is provided when the bias on the detector will exceed $\sim 210 \mu \mathrm{V}$.

To observe this 'time-resolving' detection, the effect of the detector bias $V_{\mathrm{d}}$ was monitored up to $1.5 \mathrm{mV}$. Applying a high bias to the detector without modifying the transmission through the quantum dot is by no means a trivial task, as the electrostatic coupling between the detector and the quantum dot is strong ${ }^{15}$. Hence, the genuine dephasing was excluded from the inadvertent electrostatic effect by setting the detector to a regime where it is not sensitive to the potential in the quantum dot (Fig. 3b). Compensating for the electrostatic gating effect led to the dependence of the two Aharonov-Bohm harmonics on detector bias as shown in Fig. 3c. With increasing detector bias, the first-harmonic dropped monotonically; however, the second harmonic remained unaltered in a low-bias regime, altering its 
declining slope at $V_{\mathrm{d}} \sim 500 \mu \mathrm{V}$. Still the suppression rate of the second Aharonov-Bohm harmonic remained lower than that of the first Aharonov-Bohm harmonic.

Because of the low transmission probability through the quantum dot, the major contribution of the first harmonic comes from the two direct paths through the interferometer. Thus, the dephasing rate re-18 $^{16}$ of the first Aharonov-Bohm harmonic can be analysed in a similar manner to that in the previous experimental work with an open-loop interferometer ${ }^{5}$. The expected visibility (in terms of its fast-Fourier-transformed (FFT) amplitude $A_{1}$ ) has the form, $v=A_{\mathrm{l}} / A_{1}\left(V_{\mathrm{d}}=0\right)=1-\Gamma_{\mathrm{d}} / \Gamma_{\mathrm{e}}$, where $\Gamma_{\mathrm{e}}$ is the natural broadening of the state in the quantum dot due to coupling to the leads, and $\Gamma_{\mathrm{d}}$ is the dephasing rate induced by the charge detection. $\Gamma_{\mathrm{e}} \sim 0.33 \mu \mathrm{eV}$ in our experiment ${ }^{7}$ and $\Gamma_{\mathrm{d}}$ is given by an algebraic sum of two different contributions $\Gamma_{\mathrm{d}}=\Gamma_{\mathrm{T}}+\Gamma_{\phi}$, where $\Gamma_{\mathrm{T}}$ and $\Gamma_{\phi}$ correspond to the current- and the phase-sensitive dephasing rates, respectively ${ }^{19-24}$, expressed as:

$$
\begin{gathered}
\Gamma_{\mathrm{T}}=\left(e V_{\mathrm{d}} / 8 \pi\right)\left(\Delta T_{\mathrm{d}}\right)^{2} / T_{\mathrm{d}}\left(1-T_{\mathrm{d}}\right), \\
\Gamma_{\phi}=\left(e V_{\mathrm{d}} / 2\right) T_{\mathrm{d}}\left(1-T_{\mathrm{d}}\right)(\Delta \phi)^{2} .
\end{gathered}
$$

The phase sensitivity, $\Delta \phi$, is defined as the relative phase shift of the transmitted and reflected partial waves induced by an extra charge in the quantum dot. Recently, an unexpectedly large dephasing rate was observed and interpreted ${ }^{15}$ only in terms of $\Gamma_{\mathrm{T}}$. It has been theoretically proposed that this can be understood by taking into account $\Gamma_{\phi}$ as well, which is much larger than $\Gamma_{\mathrm{T}}$ in a generic situation with a non-negligible asymmetry in the charge response of the QPC potential ${ }^{21}$. In practice, $\Delta \phi$ cannot be directly extracted from our measurement set-up. The best fit to the data for the first Aharonov-Bohm harmonic, with $\Delta \phi(=0.031)$ as a fitting parameter, is given by the solid line in Fig. $3 \mathrm{c}$. The fits reveal that the phase-sensitive dephasing mechanism is more effective than that of the current-sensitive dephasing (namely, $\Gamma_{\phi} / \Gamma_{\mathrm{T}} \sim 35$ ), which is consistent with the previous observation ${ }^{15,21}$.

The question of why the dephasing rate of the second Aharonov-Bohm harmonic at higher detector bias is so low may still be raised. We speculate that the lower rate may originate from the finite size of the electron wave packet in the detector channel, being larger than the interferometer. The time-resolving detection is effective only when the size of the electron wave packets is infinitesimally small. The dephasing can be alternatively understood in terms of the back-action ${ }^{3}$, which is the randomization of the phase of an electron passing through the quantum dot due to the fluctuations of the quantum dot potential induced by the current noise in the QPC detector ${ }^{3}$. For the trajectory in Fig. $1 \mathrm{~b}$ (first-harmonic interference), the random phase is collected for the right path during the entire passage of a wave packet through the quantum dot, whereas no random phase is collected for the left path, which leads to the suppression of the first-harmonic interference. However, for the trajectory in Fig. 1c (second-harmonic interference), both wave packets taking the left and the right paths dwell some time in the quantum dot simultaneously and collect a common random phase, which does not suppress the interference. The random phase collected while only one of the packets occupies the quantum dot suppresses the interference. Thus, the random phase accumulated for the second harmonic should be smaller than that of the first harmonic, which gives a qualitative explanation for the lower dephasing rate of the second harmonic.

So far, it has been widely accepted that the quantum mechanical complementarity of a particle can be understood in terms of the momentum transfer (or back-action), which is inevitably caused by detecting the path of the particle, as explicitly stated by Feynman et al. ${ }^{2}$ a few decades ago. Recently, however, it has been demonstrated that the particle-like behaviour also takes place by the which-path information even for the sufficiently weak momentum transfer ${ }^{5}$, which refutes the back-action picture of the dephasing. In clear contrast to previous studies, our work confirms that the wave-like behaviour is preserved unless the which-path information can be acquired out of the detection process, even if it can be done only in principle, regardless of the strength of the finite 'disturbance' caused by the charge detection. This is verified by investigating the second-harmonic dephasing in a closed-loop Aharonov-Bohm-ring interferometer, which has no analogue in the systems studied previously, including the optical ${ }^{4}$, atomic ${ }^{5}$ and solid-state $^{6}$ interferometers. Thus, this study demonstrates that acquisition of which-path information is of prime importance for quantum mechanical complementarity.

\section{METHODS}

The 2DEG in a GaAs/AlGaAs heterojunction wafer resided about $80 \mathrm{~nm}$ below the surface. The electron density was $n_{\mathrm{s}}=1.8 \times 10^{11} \mathrm{~cm}^{-2}$ with a corresponding mobility of $\mu=3.3 \times 10^{6} \mathrm{~cm}^{2} \mathrm{~V}^{-1} \mathrm{~s}^{-1}$ at $4.2 \mathrm{~K}$, resulting in an elastic mean free path of $l_{\mathrm{e}} \sim 20 \mu \mathrm{m}$. Figure 1a shows a scanning electron micrograph of the device used in this study. Negative voltages were applied to the gates so as to pinch off the 2DEG underneath them. Four side gates $\left(\mathrm{M}_{1}, \mathrm{M}_{2}, \mathrm{M}_{3}, \mathrm{M}_{4}\right)$ together with an island gate $(\mathrm{P})$ defined an Aharonov-Bohm ring with a geometrical radius of about $550 \mathrm{~nm}$, which was also confirmed by the Aharonov-Bohm interference oscillations. Two QPCs $\left(\mathrm{Q}_{1}, \mathrm{Q}_{2}\right)$ at the source and the drain were used to configure the two-terminal measurement. A quantum dot and a QPC detector were placed to detect charges trapped inside the quantum dot. Two gates $\left(\mathrm{F}_{1}, \mathrm{~F}_{2}\right)$ defined the quantum dot and separated it from the QPC detector. The island gate was electrically controlled through the bridged electrode P. The measurements were made by applying a $10 \mu \mathrm{V}$ r.m.s. excitation voltage to the source and monitoring the output current at the drain (the electron temperature was $140 \mathrm{mK}$ ).

Received 18 September 2007; accepted 20 December 2007; published 3 February 2008.

References

1. Bohr, N. in Quantum Theory and Measurement (eds Wheeler, J. A. \& Zurek, W. H.) 9-49 (Princeton Univ. Press, Princeton, 1983).

2. Feynman, R., Leighton, R. \& Sands, M. The Feynman Lectures on Physics Vol. III, Ch. 1 (Addison Wesley, Reading, 1965).

3. Stern, A., Aharonov, Y. \& Imry, Y. Phase uncertainty and loss of interference: A general picture. Phys. Rev. A 41, 3436-3448 (1990).

4. Zou, X. Y., Wang, L. J. \& Mandel, L. Induced coherence and indistinguishability in optical interference. Phys. Rev. Lett. 67, 318-321 (1991).

5. Dürr, S., Nonn, T. \& Rempe, G. Origin of quantum-mechanical complementarity probed by a 'which-way' experiment in an atom interferometer. Nature 395, 33-37 (1998).

6. Buks, E., Schuster, R., Heiblum, M., Mahalu, D. \& Umansky, V. Dephasing in electron interference by a 'which-path' detector. Nature 391, 871-874 (1998).

7. Yacoby, A., Heiblum, M., Mahalu, D. \& Shtrikman, H. Coherence and phase sensitive measurements in a quantum dot. Phys. Rev. Lett. 74, 4047-4050 (1995).

8. Schuster, R. et al. Phase measurement in a quantum dot via a double-slit interference experiment Nature 385, 417-420 (1997).

9. Field, M. et al. Measurements of Coulomb blockade with a noninvasive voltage probe. Phys. Rev. Lett. 70, 1311-1314 (1993).

10. Sprinzak, D., Ji, Y., Heiblum, M., Mahalu, D. \& Shtrikman, H. Charge distribution in a Kondo-correlated quantum dot. Phys. Rev. Lett. 88, 176805 (2002).

11. Avinun-Kalish, M., Heiblum, M., Zarchin, O., Mahalu, D. \& Umansky, V. Crossover from 'mesoscopic' to 'universal' phase for electron transmission in quantum dots. Nature $\mathbf{4 3 6}$ 529-533 (2005).

12. Khym, G. L. \& Kang, K. Charge detection in a closed-loop Aharonov-Bohm interferometer. Phys. Rev. B 74, 153309 (2006).

13. Aharonov, Y. \& Bohm, D Significance of electromagnetic potentials in the quantum theory. Phy. Rev. 115, 485-491 (1959).

14. Aronov, A. G. \& Sharvin, Yu. V. Magnetic flux effects in disordered conductors. Rev. Mod. Phys. 59, 755-779 (1987).

15. Avinun-Kalish, M., Heiblum, M., Silva, A., Mahalu, D. \& Umansky, V. Controlled dephasing of a quantum dot in the Kondo regime. Phys. Rev. Lett. 92, 156801 (2004).

16. Aleiner, I. L., Wingreen, N. S. \& Meir, Y. Dephasing and the orthogonality catastrophe in tunneling through a quantum dot: "The which path?" interferometer. Phys. Rev. Lett. 79 3740-3743 (1997).

17. Gurvitz, S. A. Measurements with a noninvasive detector and dephasing mechanism. Phys. Rev. B 56, 15215-15223 (1997).

18. Levinson, Y. Dephasing in a quantum dot due to coupling with a quantum point contact. Europhys. Lett. 39, 299-304 (1997).

19. Stodolsky, L. Measurement process in a variable-barrier system. Phys. Lett. B 459, 193-200 (1999).

20. Sprinzak, D., Buks, E., Heiblum, M. \& Shtrikman, H. Controlled dephasing of electrons via a phase sensitive detector. Phys. Rev. Lett. 84, 5820-5823 (2000). 
21. Kang, K. Decoherence of the Kondo singlet via a quantum point contact detector. Phys. Rev. Lett. 95, 206808 (2005).

22. Buttiker, M. \& Martin, A. M. Charge relaxation and dephasing in Coulomb-coupled conductors. Phys. Rev. B 61, 2737-2741 (2000).

23. Levinson, Y. Quantum dot dephasing by edge states. Phys. Rev. B 61, 4748-4753 (2000).

24. Hackenbroich, G. Phase coherent transmission through interacting mesoscopic systems. Phys. Rep. 343, 463-538 (2001)

\section{Acknowledgements}

H.-J.L. was supported by the Electron Spin Science Center in POSTECH and the Pure Basic Research

Program (Grant No. R01-2006-000-11248-0) administered by the Korea Science and Engineering
Foundation (KOSEF), by the Korea Research Foundation (Grant No. KRF-2005-070-C00055) and by the POSTECH Core Research Program. Y.C. was supported by the Korea Foundation for International Cooperation of Science and Technology (KICOS; Grant No. 2006-04969), Nanoscopia Center of Excellence (NCoE; Grant No. M60504000249-06A0400-24910) at Hanyang University through a grant provided by the Korean Ministry of Science \& Technology, and the Priority Research Centers Program (Grant No. KRF-2006-005-J02801) funded by KRF. K.K. was also supported by KRF (Grant No. KRF-2006-331-C00016). M.H. wishes to acknowledge the partial support of the
MINERVA foundation, the German Israeli foundation (GIF), the German Israeli project cooperation MINERVA foundation, the German Israeli foundation (GIF), the German Israeli project cooperation
(DIP), the Israeli Science foundation (ISF) and the Korea Ministry of Science and Technology program. Correspondence and requests for materials should be addressed to Y.C. or H.-J.L.

Reprints and permission information is available online at http://npg.nature.com/reprintsandpermissions/ 